\title{
HEAT KERNEL LOWER GAUSSIAN ESTIMATES IN THE DOUBLING SETTING WITHOUT POINCARÉ INEQUALITY
}

\author{
SAlahaddine Boutayeb
}
Abstract
In the setting of a manifold with doubling property satisfying a Gaussian upper estimate of the heat kernel, one gives a character- ization of the lower Gaussian estimate in terms of certain Hölder inequalities.

\section{CONTENTS}

Introduction $\quad 457$

1. Assumptions 461

2. Preliminaries 462

3. Hölder continuity of the heat kernel 462

4. Characterization of the lower gaussian estimate by some Hölder inequalities $\quad 466$

4.1. First characterization 466

4.2. Second characterization 470

4.3. Further results $\quad 475$

$\begin{array}{ll}\text { References } & 477\end{array}$

\section{Introduction}

Let $M$ be a connected non-compact Riemannian manifold without boundary, and let $\Delta$ denote the Laplace-Beltrami operator on $M$. Consider the heat equation

$$
\frac{\partial u}{\partial t}-\Delta u=0
$$

where $u=u(x, t), x \in M, t>0$.

2000 Mathematics Subject Classification. Primary: 58J35; Secondary: 47D07, $46 \mathrm{E} 35$.

Key words. Heat kernel, Hölder inequalities. 
The heat kernel $p_{t}(x, y)$ is by definition the smallest positive fundamental solution to the heat equation on $] 0,+\infty[\times M$. In particular, the Cauchy problem

$$
\left\{\begin{array}{l}
\frac{\partial u}{\partial t}=\Delta u \\
u(0, .)=f(.)
\end{array}\right.
$$

where $f$ is a bounded continuous function, is solved by

$$
u(t, x)=\int_{M} p_{t}(x, y) f(y) d y .
$$

In the Euclidean space $\mathbb{R}^{n}$, the heat kernel is given by the following well known formula:

$$
p_{t}(x, y)=\frac{1}{(4 \pi t)^{n / 2}} \exp \left(-\frac{|x-y|^{2}}{4 t}\right) .
$$

Denote by $B(x, r)$ the ball of center $x \in M$ and radius $r>0$ with respect to the Riemannian distance $d$, and by $V(x, r)$ its Riemannian volume. If $M$ is geodesically complete and has non-negative Ricci curvature, Li and Yau (see [14]) found that the heat kernel satisfies the two-sided Gaussian estimate:

$$
\begin{array}{r}
\frac{c}{V(x, \sqrt{t})} \exp \left(-\frac{d^{2}(x, y)}{c t}\right) \leq p_{t}(x, y) \leq \frac{C}{V(x, \sqrt{t})} \\
\exp \left(-\frac{d^{2}(x, y)}{C t}\right) \\
\forall t>0, x, y \in M
\end{array}
$$

One says that $M$ satisfies the doubling property if

$$
V(x, 2 r) \leq C V(x, r), \quad \forall x \in M, r>0 .
$$

A consequence of $(D)$ is that there exists $\nu>0$ such that

$$
\frac{V(x, s)}{V(x, r)} \leq C\left(\frac{s}{r}\right)^{\nu}, \quad \forall x \in M, s \geq r>0 .
$$

Note that, if $\operatorname{Ricc} M \geq 0$, then $M$ Satisfies the doubling property. We say that $M$ admits the relative Faber-Krahn inequality if for any ball $B(x, r) \subset M$ and any precompact open set $\Omega \subset B(x, r)$

$$
\lambda_{1}(\Omega) \geq \frac{b}{r^{2}}\left(\frac{V(x, r)}{\mu(\Omega)}\right)^{\nu},
$$

where $\lambda_{1}(\Omega)$ is the first Dirichlet eigenvalue for $-\Delta, b$ and $\nu$ some positive constants. 
On a geodesically complete manifold (see [9]), Grigor'yan proved that $(F K)$ is equivalent to the Gaussian upper estimate

$(U E) \quad p_{t}(x, y) \leq \frac{C}{V(x, \sqrt{t})} \exp \left(-\frac{d^{2}(x, y)}{C t}\right), \quad t>0, x, y \in M$,

in conjunction with the doubling property $\left(D_{\nu}\right)$.

Saloff-Coste (see [18], [19]) found that the two-sided Gaussian estimate of the heat kernel

$$
\begin{array}{r}
\frac{c}{V(x, \sqrt{t})} \exp \left(-\frac{d^{2}(x, y)}{c t}\right) \leq p_{t}(x, y) \leq \frac{C}{V(x, \sqrt{t})} \exp \left(-\frac{d^{2}(x, y)}{C t}\right) \\
t>0, x, y \in M
\end{array}
$$

is equivalent to $(D)$ and the 2-Poincaré inequalities

$\left(P_{2}\right) \quad \int_{B(x, r)}\left|f(y)-f_{r}(x)\right|^{2} d \mu(y) \leq C r^{2} \int_{B(x, C r)}|\nabla f(y)|^{2} d \mu(y)$,

for all $f \in \mathcal{C}_{0}^{\infty}(M), x \in M, r>0$, where $f_{r}(x)=\frac{1}{V(x, r)} \int_{B(x, r)} f(y) d \mu(y)$, see also $[8]$.

Assuming that the Gaussian upper estimate holds, Coulhon and Ouhabaz gave some simple inequalities (other than Poincaré) that are necessary/sufficient to complete the two-sided Gaussian estimate.

In the case where the volume growth is polynomial, that is

$$
V(x, r) \simeq r^{D}, \quad D>0,
$$

Coulhon (see [5]) proved that, if $(U E)$ holds, then

$$
\frac{c}{V(x, \sqrt{t})} \exp \left(-\frac{d^{2}(x, y)}{c t}\right) \leq p_{t}(x, y), \quad t>0, x, y \in M,
$$

is equivalent to the Sobolev type inequality

$$
\left(S^{D}\right) \quad \frac{|f(x)-f(y)|}{d(x, y)^{\alpha-(D / p)}} \leq C\left\|\Delta^{\alpha / 2} f\right\|_{p}, \quad \forall f \in \mathcal{C}_{0}^{\infty}(M), x, y \in M,
$$

for $p>1$ and some $\alpha>\frac{D}{p}$.

In this polynomial setting and assuming $(U E)$, Ouhabaz (see $[\mathbf{1 6}]$ ) had given previously a characterization of $(L E)$ in terms of a weaker Gagliardo-Nirenberg type inequality:

$\left(G^{D}\right) \quad \frac{|f(x)-f(y)|}{[d(x, y)]^{\alpha-(D / p)}} \leq C\|f\|_{p}^{1-\theta}\left\|\Delta^{\alpha / 2 \theta} f\right\|_{p}^{\theta}, \quad x, y \in M$,

for $p>1$ and some $\left.\alpha>\frac{D}{p}, \theta \in\right] 0,1[$. 
In the more general case when the doubling condition $\left(D_{\nu}\right)$ is satisfied, Coulhon proved that, if $(U E)$ holds, then for any $p>1$ and $\alpha>\frac{\nu}{p}$ :

$$
|f(x)-f(y)| \leq C \frac{d^{\alpha}(x, y)}{V^{1 / p}(x, d(x, y))}\left\|\Delta^{\alpha / 2} f\right\|_{p},
$$

implies $(L E)$.

The aim of the present paper is to give similar necessary and sufficient conditions for the lower Gaussian estimate $(L E)$ in the general setting when the volume is only doubling. More precisely, the necessary/sufficient conditions will be given by the following main results:

Theorem. Assume that $\left(D_{\nu}\right)$ and $(U E)$ holds. Then

$$
\frac{c}{V(x, \sqrt{t})} \exp \left(-\frac{d^{2}(x, y)}{c t}\right) \leq p_{t}(x, y), \quad t>0, x, y \in M,
$$

implies that for all p large enough there are $\alpha, \alpha^{\prime}>\frac{\nu}{p}$ such that

$(S)\left\{\begin{array}{l}|f(x)-f(y)| \leq \frac{C}{V^{1 / p}(x, d)} \max \left\{d^{\alpha}\left\|\Delta^{\alpha / 2} f\right\|_{p}, d^{\alpha^{\prime}}\left\|\Delta^{\alpha^{\prime} / 2} f\right\|_{p}\right\} \\ \forall f \in \mathcal{D}, x, y \in M\end{array}\right.$

Conversely, $(S)$ for any $p>1$ and $\alpha, \alpha^{\prime}>\frac{\nu}{p}$, implies (LE), where $d$ denote to $d(x, y)$.

Theorem. Assume that $\left(D_{\nu}\right)$ and $(U E)$ holds. Then

$$
\frac{c}{V(x, \sqrt{t})} \exp \left(-\frac{d^{2}(x, y)}{c t}\right) \leq p_{t}(x, y), \quad t>0, x, y \in M
$$

implies for all $p$ large enough, there are $\alpha, \alpha^{\prime}>\frac{\nu}{p}$ and $\left.\theta, \theta^{\prime} \in\right] 0,1[$ such that $\alpha-\alpha^{\prime}=\frac{\nu}{p}, \frac{\alpha}{\theta}=\frac{\alpha^{\prime}}{\theta^{\prime}}$ and

$$
\left\{\begin{aligned}
\mid f(x) & -f(y) \mid \leq \frac{C}{V^{1 / p}(x, d)} \\
& \times \max \left\{d^{\alpha}\|f\|_{p}^{1-\theta}\left\|\Delta^{\alpha / 2 \theta} f\right\|_{p}^{\theta}, d^{\alpha^{\prime}}\|f\|_{p}^{1-\theta^{\prime}}\left\|\Delta^{\alpha^{\prime} / 2 \theta^{\prime}} f\right\|_{p}^{\theta^{\prime}}\right\} \\
\forall f \in \mathcal{D}, x, y \in M &
\end{aligned}\right.
$$

Conversely, $(G)$ for any $p>1, \alpha, \alpha^{\prime}>\frac{\nu}{p}$ and $\left.\theta, \theta^{\prime} \in\right] 0,1[$, implies $(L E)$, where $d$ denote to $d(x, y)$.

There are several classes of fractal spaces which satisfy the more general form of the heat kernel estimate:

$$
\frac{c}{V\left(x, t^{1 / w}\right)} E_{w}(c, x, y, t) \leq p_{t}(x, y) \leq \frac{C}{V\left(x, t^{1 / w}\right)} E_{w}(C, x, y, t),
$$


for $t>0, x, y \in M$, where

$$
E_{w}(\lambda, x, y, t)=\exp \left(-\left(\frac{d^{w}(x, y)}{\lambda t}\right)^{1 /(w-1)}\right), \quad \forall t, \lambda>0, x, y \in M,
$$

and $w$ is a so-called escape time or random walk dimension (see [1], [2], $[\mathbf{3}]$, see also $[\mathbf{1 0}],[\mathbf{1 1}],[\mathbf{1 2}])$. We are also able to write our characterizations of lower heat kernel estimate in this general form.

We choose to write this paper in a more general setting, when $M$ is a metric measure space endowed with a symetric Markov semigroup.

\section{Assumptions}

In the sequel, we shall place ourselves in a setting similar to the one in [5], $[\mathbf{1 0}],[\mathbf{1 3}]$ : Let $(M, d, \mu)$ be a metric measure space endowed with a symmetric Markov semigroup $e^{-t A}$ on $L^{2}(M, \mu)$ with a measurable kernel $p_{t}$, that is

$e^{-t A} f(x)=\int_{M} p_{t}(x, y) f(y) d \mu(y), \quad t>0, f \in L^{2}(M, \mu), \mu$-a.e. $x \in M$.

The powers $A^{\alpha}, \alpha>0$, of the operator $A$ are densely defined on $L^{p}(M, \mu)$, $1 \leq p<+\infty$, we denote by $\mathcal{D}_{p}\left(A^{\alpha}\right)$ their domain. Background and more information about this functional setting can be found in $[\mathbf{1 7}]$ and references therein. For simplicity, we always write $\mathcal{D}$ for the space $\mathcal{D}_{p}\left(A^{\alpha}\right)$ required by the context. We assume that $\left\|e^{-t A} f\right\|_{p} \rightarrow 0$ as $t \rightarrow+\infty$, for all $f \in \mathcal{D}$ and $1 \leq p<+\infty$.

We shall assume that for all $x \in M$ and $r>0,0<V(x, r)<+\infty$ and that the metric space $(M, d)$ satisfies the chain condition: there exists $C>0$ such that, for all $x, y \in M$, for any $n \in \mathbb{N}^{*}$, there exists a sequence $\left\{x_{i}\right\}_{i=0}^{n}$ of points in $M$ such that $x_{0}=x, x_{n}=y$ and

$$
d\left(x_{i}, x_{i+1}\right) \leq C \frac{d(x, y)}{n}, \quad \forall i=0, \ldots, n-1 .
$$

We say that such a sequence is a chain connecting $x$ and $y$.

Note that these assumptions are well satisfied in the setting of the introduction when $M$ is a connected non-compact complete Riemannian manifold. They will be standing assumptions in this paper and we will refer to them by writing: let $(M, d, \mu, A)$ be as above. We recall that a symmetric Markov semigroup on $L^{2}(M, \mu)$ is analytic on $L^{p}(M, \mu)$ (see [21]). 


\section{Preliminaries}

\section{Notation:}

Consider a parameter $w \geq 2$,

$$
\begin{aligned}
& \left(U E_{w}\right) \quad p_{t}(x, y) \leq \frac{C}{V\left(x, t^{1 / w}\right)} E_{w}(C, x, y, t), \quad \forall t>0, x, y \in M . \\
& \left(L E_{w}\right) \quad \frac{c}{V\left(x, t^{1 / w}\right)} E_{w}(c, x, y, t) \leq p_{t}(x, y), \quad \forall t>0, x, y \in M . \\
& \left(D L E_{w}\right) \quad \frac{c}{V\left(x, t^{1 / w}\right)} \leq p_{t}(x, x), \quad \forall t>0, x \in M . \\
& \left(L Y_{w}\right) \quad \frac{c}{V\left(x, t^{1 / w}\right)} E_{w}(c, x, y, t) \leq p_{t}(x, y) \leq \frac{C}{V\left(x, t^{1 / w}\right)} E_{w}(C, x, y, t),
\end{aligned}
$$

for all $t>0$ and $x, y \in M$.

Note that $\left(U E_{2}\right)=(U E)$ and similarly for the others $w$-estimations. For all $p>1$, we denote by $p^{\prime}$ the conjugate exponent of $p, \frac{1}{p}+\frac{1}{p^{\prime}}=1$. Letters $c, C, C^{\prime}$ are normally used to denote unimportant positive constants, whose values may change at each occurence. In the sequel, for the sake of simplicity, we sometimes denote $d(x, y)$ by $d$.

We recall the following proposition. for the proof, in the case $w=2$, see [4, Lemma 1, p. 224], [7, §6] or [20, Theorem 4.2.8], [13, §3.3]; for the general case $w \geq 2$, see $[\mathbf{1 6}, \S 4]$ or $[\mathbf{5}, \S 3]$.

Proposition 1. Assume that $(M, d, \mu)$ satisfies the doubling property. If $\left(U E_{w}\right)$ holds, then $\left(D L E_{w}\right)$ holds.

The following lemma follows from [10, Corollary 3.5], [13, Lemma 5.1], see also [5, p. 801].

Lemma 2. Assume that $(M, d, \mu)$ satisfies the doubling property. If $\left(U E_{w}\right)$ holds and there exist $a, c>0$ such that

$$
\frac{c}{V\left(x, t^{1 / w}\right)} \leq p_{t}(x, y), \text { for all } t>0, x, y \in M \text { with } d(x, y) \leq a t^{1 / w}
$$

then $\left(L E_{w}\right)$ holds.

\section{Hölder continuity of the heat kernel}

At first, let us recall the following proposition, which follows from $[\mathbf{1 9}$, Proposition 3.2] in the case $w=2$ and $[\mathbf{1 3}, \S 5.3]$ for the general case. 
Proposition 3. Assume that $(M, d, \mu)$ satisfies the doubling property. If $\left(L Y_{w}\right)$ holds, then there exists $\left.\eta \in\right] 0,1[$ and $C>0$ such that

$$
\begin{aligned}
& \left|p_{t}(x, z)-p_{t}(y, z)\right| \leq \frac{C}{V\left(z, t^{1 / w}\right)}\left(\frac{d(x, y)}{t^{1 / w}}\right)^{\eta}, \\
& \qquad \text { for all } t>0 \text { and } \mu \text {-a.e. } x, y, z \in M .
\end{aligned}
$$

In the next result, we give an estimation with a Gaussian factor.

Proposition 4. Assume that $(M, d, \mu)$ satisfies the doubling condition $\left(D_{\nu}\right)$. If $\left(L Y_{w}\right)$ holds, then there exists $\left.\eta \in\right] 0,1[$ and $C>0$ such that for all $p>1$ :

$$
\begin{aligned}
& \left|p_{t}(x, z)-p_{t}(y, z)\right| \\
& \leq C \frac{\left(E_{w}(C, x, z, t)+E_{w}(C, y, z, t)\right)^{1 / p}}{V^{1 / p}(x, d) V^{1 / p^{\prime}}\left(z, t^{1 / w}\right)} \begin{cases}\left(\frac{d}{t^{1 / w}}\right)^{\frac{\eta}{p^{\prime}}+\frac{\nu}{p}} & \text { if } d \geq t^{1 / w} \\
\left(\frac{d}{t^{1 / w}}\right)^{\frac{\eta}{p^{\prime}}} & \text { if not, }\end{cases}
\end{aligned}
$$

for all $t>0$ and $\mu$-a.e. $x, y, z \in M$, where $d$ denote to $d(x, y)$.

Proof: Let $x, y, z \in M, p>1$ and $p^{\prime}$ its conjugate exponent, one may write

$$
\left|p_{t}(x, z)-p_{t}(y, z)\right| \leq\left|p_{t}(x, z)-p_{t}(y, z)\right|^{1 / p^{\prime}} \cdot\left(p_{t}(x, z)+p_{t}(y, z)\right)^{1 / p} .
$$

According to Proposition 3 and $\left(U E_{w}\right)$, it follows

$$
\text { (1) } \begin{aligned}
\left|p_{t}(x, z)-p_{t}(y, z)\right| \leq \frac{C}{V^{1 / p^{\prime}}\left(z, t^{1 / w}\right)}\left(\frac{d(x, y)}{t^{1 / w}}\right)^{\frac{\eta}{p^{\prime}}} \\
\times\left(\frac{1}{V\left(x, t^{1 / w}\right)} E_{w}(C, x, z, t)+\frac{1}{V\left(y, t^{1 / w}\right)} E_{w}(C, y, z, t)\right)^{1 / p} .
\end{aligned}
$$

Denote to $d(x, y)$ by $d$. Since $V(x, d) \leq V(y, 2 d)$ and $V(y, 2 d) \leq C V(y, d)$, then $V(x, d) \leq C V(y, d)$.

Assume that $d \geq t^{1 / w}$. From $\left(D_{\nu}\right)$, one has

$$
\frac{1}{V\left(x, t^{1 / w}\right)} \leq \frac{C}{V(x, d)}\left(\frac{d}{t^{1 / w}}\right)^{\nu}
$$

and

$$
\frac{1}{V\left(y, t^{1 / w}\right)} \leq \frac{C}{V(y, d)}\left(\frac{d}{t^{1 / w}}\right)^{\nu} \leq \frac{C^{\prime}}{V(x, d)}\left(\frac{d}{t^{1 / w}}\right)^{\nu}
$$


Then, (1) yields

$$
\begin{aligned}
\left|p_{t}(x, z)-p_{t}(y, z)\right| \leq \frac{C}{V^{1 / p}(x, d)} & \left(\frac{d}{t^{1 / w}}\right)^{\frac{\eta}{p^{\prime}}+\frac{\nu}{p}} \frac{1}{V^{1 / p^{\prime}}\left(z, t^{1 / w}\right)} \\
\times & {\left[E_{w}(C, x, z, t)+E_{w}(C, y, z, t)\right]^{1 / p} . }
\end{aligned}
$$

If $d \leq t^{1 / w}$. One has also

$$
\frac{1}{V\left(y, t^{1 / w}\right)} \leq \frac{1}{V(y, d)} \leq \frac{C}{V(x, d)}
$$

Analogously, by (1) it follows that

$$
\begin{aligned}
\left|p_{t}(x, z)-p_{t}(y, z)\right| \leq \frac{C}{V^{1 / p}(x, d)} & \left(\frac{d}{t^{1 / w}}\right)^{\frac{\eta}{p^{\prime}}} \frac{1}{V^{1 / p^{\prime}}\left(z, t^{1 / w}\right)} \\
& \times\left[E_{w}(C, x, z, t)+E_{w}(C, y, z, t)\right]^{1 / p} .
\end{aligned}
$$

Now, we derive an oscillation estimate for the semigroup $\left(e^{-t A}\right)_{t>0}$.

Proposition 5. Assume that $(M, d, \mu)$ satisfies the doubling condition $\left(D_{\nu}\right)$. If $\left(L Y_{w}\right)$ holds, then for all $p>1$ there exists $C>0$ such that

$$
\begin{aligned}
& \mid e^{-t A} f(x)- e^{-t A} f(y) \mid \\
& \leq \frac{C\|f\|_{p}}{V^{1 / p}(x, d(x, y))} \begin{cases}\left(\frac{d(x, y)}{t^{1 / w}}\right)^{\frac{\eta}{p^{\prime}}+\frac{\nu}{p}} & \text { if } d(x, y) \geq t^{1 / w} \\
\left(\frac{d(x, y)}{t^{1 / w}}\right)^{\frac{\eta}{p^{\prime}}} & \text { if not },\end{cases}
\end{aligned}
$$

for all $t>0, f \in \mathcal{D}$ and $\mu$-a.e. $x, y \in M$.

Proof: From the definition of the heat kernel, one can write

$$
\left|e^{-t A} f(x)-e^{-t A} f(y)\right| \leq \int_{M}\left|\left(p_{t}(x, z)-p_{t}(y, z)\right) f(z)\right| d \mu(z),
$$

for all $t>0, f \in \mathcal{D}, x, y \in M$. 
Assume that $d \geq t^{1 / w}$. From Proposition 4 , it follows

$$
\begin{aligned}
& \int_{M}\left|\left(p_{t}(x, z)-p_{t}(y, z)\right) f(z)\right| d \mu(z) \leq \frac{C}{V^{1 / p}(x, d)}\left(\frac{d}{t^{1 / w}}\right)^{\frac{\eta}{p^{\prime}}+\frac{\nu}{p}} \\
& \quad \times \int_{M} \frac{|f(z)|}{V^{1 / p^{\prime}}\left(z, t^{1 / w}\right)}\left(E_{w}(C, x, z, t)+E_{w}(C, y, z, t)\right)^{1 / p} d \mu(z),
\end{aligned}
$$

and by using Hölder inequality, we obtain

$$
\text { (2) } \begin{aligned}
& \int_{M}\left|\left(p_{t}(x, z)-p_{t}(y, z)\right) f(z)\right| d \mu(z) \leq \frac{C}{V^{1 / p}(x, d)}\left(\frac{d}{t^{1 / w}}\right)^{\frac{\eta}{p^{\prime}}+\frac{\nu}{p}}\|f\|_{p} \\
& \times\left(\int_{M} \frac{1}{V\left(z, t^{1 / w}\right)}\left(E_{w}(C, x, z, t)+E_{w}(C, y, z, t)\right)^{p^{\prime} / p} d \mu(z)\right)^{1 / p^{\prime}} .
\end{aligned}
$$

Let us prove that for all $\gamma>0$, there is $C>0$, such that for all $t>0$.

$$
\int_{M} \frac{1}{V\left(z, t^{1 / w}\right)}\left(E_{w}(C, x, z, t)+E_{w}(C, y, z, t)\right)^{\gamma} d \mu(z) \leq C .
$$

Indeed, since $(a+b)^{\gamma} \leq C_{\gamma}\left(a^{\gamma}+b^{\gamma}\right)$, for all $a \geq 0$ and $b \geq 0$, then

$$
\begin{aligned}
\left(E_{w}(C\right. & \left., x, z, t)+E_{w}(C, y, z, t)\right)^{\gamma} \\
& \leq C\left(E_{w}^{\gamma}(C, x, z, t)+E_{w}^{\gamma}(C, y, z, t)\right) \\
& \leq C\left(E_{w}\left(C \gamma^{1-w}, x, z, t\right)+E_{w}\left(C \gamma^{1-w}, y, z, t\right)\right)
\end{aligned}
$$

Let $c$ be the constant in $\left(L E_{w}\right)$. Set $a=\frac{c}{C \gamma^{1-w}}$ and $s=\frac{t}{a}$, then

$$
\begin{aligned}
E_{w}\left(C \gamma^{1-w}, x, z, t\right) & =E_{w}(c / a, x, z, a s) \\
& =E_{w}(c, x, z, s) .
\end{aligned}
$$

Therefore

$$
\begin{aligned}
& \int_{M} \frac{1}{V\left(z, t^{1 / w}\right)}\left(E_{w}(C, x, z, t)+E_{w}(C, y, z, t)\right)^{\gamma} d \mu(z) \\
& \quad \leq \int_{M} \frac{C}{V\left(z,(a s)^{1 / w}\right)}\left(E_{w}(c, x, z, s)+E_{w}(c, y, z, s)\right) d \mu(z) .
\end{aligned}
$$

By $\left(D_{\nu}\right)$, one has $V(z, \sqrt{a s}) \simeq V(z, \sqrt{s}), s>0$. On the other hand, according to $\left(L E_{w}\right)$ and since $\left(e^{-t A}\right)_{t>0}$ is Markovian, one has

$$
\int_{M} \frac{1}{V(z, \sqrt{s})} E_{w}(c, x, z, t) d \mu(z) \leq \int_{M} p_{s}(x, z) d \mu(z) \leq 1,
$$

for all $s>0$ and $x \in M$. Therefore, we deduce (3). 
By choosing $\gamma=p^{\prime} / p$, (2) yields

$$
\left|e^{-t A} f(x)-e^{-t A} f(y)\right| \leq \frac{C}{V^{1 / p}(x, d)}\left(\frac{d}{t^{1 / w}}\right)^{\frac{\eta}{p^{\prime}}+\frac{\nu}{p}}\|f\|_{p} .
$$

If $d \leq t^{1 / w}$. Using Proposition 4 and arguing as before, it follows

$$
\left|e^{-t A} f(x)-e^{-t A} f(y)\right| \leq \frac{C}{V^{1 / p}(x, d)}\left(\frac{d}{t^{1 / w}}\right)^{\frac{\eta}{p^{\prime}}}\|f\|_{p}
$$

\section{Characterization of the lower gaussian estimate by some Hölder inequalities}

\subsection{First characterization.}

Theorem 6. Let $(M, d, \mu, A)$ as above satisfy the doubling condition $\left(D_{\nu}\right)$. Let $w \geq 2$. Assume that $\left(U E_{w}\right)$ holds. Then

$\left(L E_{w}\right) \quad \frac{c}{V\left(x, t^{1 / w}\right)} E_{w}(c, x, y, t) \leq p_{t}(x, y), \quad \forall t>0, \forall x, y \in M$,

implies that for all $p$ large enough there are $\alpha, \alpha^{\prime}>\frac{\nu}{p}$ such that

$\left(S_{w}\right)\left\{\begin{array}{l}|f(x)-f(y)| \leq \frac{C}{V^{1 / p}(x, d)} \max \left\{d^{\alpha}\left\|A^{\alpha / w} f\right\|_{p}, d^{\alpha^{\prime}}\left\|A^{\alpha^{\prime} / w} f\right\|_{p}\right\} \\ \forall f \in \mathcal{D}, x, y \in M .\end{array}\right.$

Conversely, $\left(S_{w}\right)$ for any $p>1$ and $\alpha, \alpha^{\prime}>\frac{\nu}{p}$, implies $\left(L E_{w}\right)$, where $d$ denote to $d(x, y)$.

Proof:

$(\Rightarrow)$ For all $f \in \mathcal{D}, t>0$, we know that $\frac{\partial^{k}}{\partial t^{k}} e^{-t A} f=A^{k} e^{-t A} f$ for all $k \in \mathbb{N}$. Then

$$
f=\sum_{i=0}^{k-1} \frac{t^{i}}{i !} A^{i} e^{-t A} f+\frac{1}{(k-1) !} \int_{0}^{t} s^{k-1} A^{k} e^{-s A} f d s, \quad \forall k \geq 1 .
$$

Since $\left(e^{-t A}\right)_{t>0}$ is analytic, then for all $p>1$

$$
\left\|A^{i} e^{-t A} f\right\|_{p}=\left\|A^{i} e^{-(t / 2) A} e^{-(t / 2) A} f\right\|_{p} \leq C t^{-i}\left\|e^{-(t / 2) A} f\right\|_{p} .
$$

On the other hand, $e^{-t A} f \rightarrow 0, \forall f \in \mathcal{D}$ when $t \rightarrow+\infty$, then for all $k \geq 1$ :

$$
f=\frac{1}{(k-1) !} \int_{0}^{+\infty} t^{k-1} A^{k} e^{-t A} f d t, \quad \forall f \in \mathcal{D} .
$$


Hence

$$
|f(x)-f(y)| \leq C_{k} \int_{0}^{+\infty} t^{k-1}\left|A^{k} e^{-t A} f(x)-A^{k} e^{-t A} f(y)\right| d s,
$$

for all $k \geq 1$, and $f \in \mathcal{D}$.

By rewriting

$$
\begin{aligned}
\mid A^{k} e^{-t A} f(x) & -A^{k} e^{-t A} f(y) \mid \\
& =\left|e^{-(t / 2) A} A^{k} e^{-(t / 2) A} f(x)-e^{-(t / 2) A} A^{k} e^{-(t / 2) A} f(y)\right|
\end{aligned}
$$

and applying Proposition 5 to $A^{k} e^{-(t / 2) A} f$, it follows

$$
\begin{aligned}
& \mid e^{-t A} f(x)-e^{-t A} f(y) \mid \\
& \leq \frac{C\left\|A^{k} e^{-(t / 2) A} f\right\|_{p}}{V^{1 / p}(x, d(x, y))} \begin{cases}\left(\frac{d(x, y)}{t^{1 / w}}\right)^{\frac{\eta}{p^{\prime}}+\frac{\nu}{p}} & \text { if } d(x, y) \geq t^{1 / w} \\
\left(\frac{d(x, y)}{t^{1 / w}}\right)^{\frac{\eta}{p^{\prime}}} & \text { if not, }\end{cases}
\end{aligned}
$$

for all $t>0$ and $\mu$-a.e. $x, y \in M$. Then

$$
\begin{aligned}
|f(x)-f(y)| \leq \frac{C}{V^{1 / p}(x, d)} & \left(d^{\frac{\eta}{p^{\prime}}+\frac{\nu}{p}} \int_{0}^{d^{w}} t^{k-\frac{1}{w}\left(\frac{\eta}{p^{\prime}}+\frac{\nu}{p}\right)-1}\left\|A^{k} e^{-(t / 2) A} f\right\|_{p} d t\right. \\
& \left.+d^{\frac{\eta}{p^{\prime}}} \int_{d^{w}}^{+\infty} t^{k-\frac{\eta}{w p^{\prime}}-1}\left\|A^{k} e^{-(t / 2) A} f\right\|_{p} d t\right) .
\end{aligned}
$$

Now, we choose $k>\frac{1}{w}\left(\frac{\eta}{p^{\prime}}+\frac{\nu}{p}\right)$ and let $\left.\delta \in\right] 0, \frac{p}{p^{\prime}} \eta[$.

One can write

$$
\left\|A^{k} e^{-(t / 2) A} f\right\|_{p}=\left\|A^{k-\frac{1}{w}\left(\frac{\eta}{p}+\frac{\nu+\delta}{p}\right)} e^{-(t / 2) A}\left(A^{\frac{1}{w}\left(\frac{\eta}{p}+\frac{\nu+\delta}{p}\right)} f\right)\right\|_{p},
$$

then by analyticity, one has

$$
\left\|A^{k} e^{-(t / 2) A} f\right\|_{p} \leq C_{1} t^{\frac{1}{w}\left(\frac{\eta}{p^{\prime}}+\frac{\nu+\delta}{p}\right)-k}\left\|A^{\frac{1}{w}\left(\frac{\eta}{p^{\prime}}+\frac{\nu+\delta}{p}\right)} f\right\|_{p} .
$$

Similarly, one also has

$$
\left\|A^{k} e^{-(t / 2) A} f\right\|_{p} \leq C_{2} t^{\frac{1}{w}\left(\frac{\eta}{p^{\prime}}-\frac{\delta}{p}\right)-k}\left\|A^{\frac{1}{w}\left(\frac{\eta}{p^{\prime}}-\frac{\delta}{p}\right)} f\right\|_{p} .
$$


Then

$$
\begin{aligned}
|f(x)-f(y)| \leq \frac{C}{V^{1 / p}(x, d)}\left(d^{\frac{\eta}{p^{\prime}}+\frac{\nu}{p}} \int_{0}^{d^{w}} t^{\frac{\delta}{w p}-1}\left\|A^{\frac{1}{w}\left(\frac{\eta}{p^{\prime}}+\frac{\nu+\delta}{p}\right)} f\right\|_{p} d t\right. \\
\\
\left.\quad+d^{\frac{\eta}{p^{\prime}}} \int_{d^{w}}^{+\infty} t^{-\frac{\delta}{w p}-1}\left\|A^{\frac{1}{w}\left(\frac{\eta}{p^{\prime}}-\frac{\delta}{p}\right)} f\right\|_{p} d t\right) .
\end{aligned}
$$

Therefore

$$
\begin{aligned}
|f(x)-f(y)| & \leq \frac{C}{V^{1 / p}(x, d)}\left(d^{\alpha}\left\|A^{\alpha / w} f\right\|_{p}+d^{\alpha^{\prime}}\left\|A^{\alpha^{\prime} / w} f\right\|_{p}\right) \\
& \leq \frac{C}{V^{1 / p}(x, d)} \max \left\{d^{\alpha}\left\|A^{\alpha / w} f\right\|_{p}, d^{\alpha^{\prime}}\left\|A^{\alpha^{\prime} / w} f\right\|_{p}\right\},
\end{aligned}
$$

where $\alpha=\frac{\eta}{p^{\prime}}+\frac{\nu+\delta}{p}>\frac{\nu}{p}, \alpha^{\prime}=\frac{\eta}{p^{\prime}}-\frac{\delta}{p}>0$ and $\left.\delta \in\right] 0, \frac{p}{p^{\prime}} \eta[$.

For $p>\frac{\nu+\eta}{\eta}$, there is $\delta_{0}>0$ such that $\left.p>\frac{\nu+\eta+\delta}{\eta}, \forall \delta \in\right] 0, \delta_{0}[$.

Therefore for $\delta \in] 0, \delta_{0}\left[\right.$, one has $\eta>\frac{\nu+\eta+\delta}{p}$, then $\eta\left(1-\frac{1}{p}\right)>\frac{\nu+\delta}{p}$, hence $\alpha^{\prime}=\frac{\eta}{p^{\prime}}-\frac{\delta}{p}>\frac{\nu}{p}$.

$(\Leftarrow)$ Let $p>1$, assume that for some $\alpha$ and $\alpha^{\prime}>\frac{\nu}{p}$ :

$\left(S_{w}\right)\left\{\begin{array}{l}|f(x)-f(y)| \leq \frac{C}{V^{1 / p}(x, d)} \max \left\{d^{\alpha}\left\|A^{\alpha / w} f\right\|_{p}, d^{\alpha^{\prime}}\left\|A^{\alpha^{\prime} / w} f\right\|_{p}\right\} \\ \forall f \in \mathcal{D}, x, y \in M .\end{array}\right.$

Let $z \in M$, by analyticity of $\left(e^{-t A}\right)_{t>0}, p_{t}(., z)$ belong to $\mathcal{D}_{p}\left(A^{\alpha / w}\right)$. Then by choosing $f=p_{t}(., z)$ in $\left(S_{w}\right)$, one has for all $x, y \in M$ :

$$
\begin{aligned}
\mid p_{t}(x, z) & -p_{t}(y, z) \mid \\
\leq & \frac{C}{V^{1 / p}(x, d)} \max \left\{d^{\alpha}\left\|A^{\alpha / w} p_{t}(., z)\right\|_{p}, d^{\alpha^{\prime}}\left\|A^{\alpha^{\prime} / w} p_{t}(., z)\right\|_{p}\right\} .
\end{aligned}
$$

By analyticity, we obtain

$$
\left\|A^{\alpha / w} p_{t}(., z)\right\|_{p}=\left\|A^{\alpha / w} e^{-(t / 2) A} p_{t / 2}(., z)\right\|_{p} \leq C t^{-\alpha / w}\left\|p_{t / 2}(., z)\right\|_{p} .
$$

Since $\left(e^{-t A}\right)_{t>0}$ is symmetric Markovian, then $\left\|p_{t / 2}(., z)\right\|_{1} \leq 1, \forall t>0$.

Then from $\left(U E_{w}\right)$ and Hölder inequality, it follows

$$
\left\|p_{t}(., z)\right\|_{p} \leq \frac{C}{\left[V\left(z, t^{1 / w}\right)\right]^{1-\frac{1}{p}}}, \quad \forall t>0
$$


and by $\left(D_{\nu}\right)$, one has

$$
\left\|p_{t / 2}(., z)\right\|_{p} \leq \frac{C}{\left[V\left(z, t^{1 / w}\right)\right]^{1-\frac{1}{p}}}
$$

then

$$
\left\|A^{\alpha / w} p_{t}(., z)\right\|_{p} \leq C \frac{t^{-\alpha / w}}{\left[V\left(z, t^{1 / w}\right)\right]^{1-\frac{1}{p}}}
$$

Therefore

$$
\begin{aligned}
& \left|p_{t}(x, z)-p_{t}(y, z)\right| \\
& \leq \frac{C}{V^{1 / p}(x, d)} \max \left\{\left(\frac{d}{t^{1 / w}}\right)^{\alpha} \frac{1}{\left[V\left(z, t^{1 / w}\right)\right]^{1-\frac{1}{p}}},\left(\frac{d}{t^{1 / w}}\right)^{\alpha^{\prime}} \frac{1}{\left[V\left(z, t^{1 / w}\right)\right]^{1-\frac{1}{p}}}\right\} \\
& \leq \frac{C}{V\left(z, t^{1 / w}\right)}\left(\frac{V\left(z, t^{1 / w}\right)}{V(x, d)}\right)^{1 / p} \max \left\{\left(\frac{d}{t^{1 / w}}\right)^{\alpha},\left(\frac{d}{t^{1 / w}}\right)^{\alpha^{\prime}}\right\} .
\end{aligned}
$$

By Proposition $1,\left(U E_{w}\right)$ and $\left(D_{\nu}\right)$ yield

$$
\left(D L E_{w}\right) \quad \frac{c}{V\left(z, t^{1 / w}\right)} \leq p_{t}(z, z), \quad \forall z \in M
$$

Thus

$\left|p_{t}(x, z)-p_{t}(y, z)\right| \leq C\left(\frac{V\left(z, t^{1 / w}\right)}{V(x, d)}\right)^{1 / p} \max \left\{\left(\frac{d}{t^{1 / w}}\right)^{\alpha},\left(\frac{d}{t^{1 / w}}\right)^{\alpha^{\prime}}\right\} p_{t}(z, z)$.

For $z=x$, one has

$\left|p_{t}(x, x)-p_{t}(y, x)\right| \leq C\left(\frac{V\left(x, t^{1 / w}\right)}{V(x, d)}\right)^{1 / p} \max \left\{\left(\frac{d}{t^{1 / w}}\right)^{\alpha},\left(\frac{d}{t^{1 / w}}\right)^{\alpha^{\prime}}\right\} p_{t}(x, x)$.

If $d \leq t^{1 / w},\left(D_{\nu}\right)$ yields

$$
\left|p_{t}(x, x)-p_{t}(y, x)\right| \leq C \max \left\{\left(\frac{d}{t^{1 / w}}\right)^{\alpha-\frac{\nu}{p}},\left(\frac{d}{t^{1 / w}}\right)^{\alpha^{\prime}-\frac{\nu}{p}}\right\} p_{t}(x, x) .
$$

Since $\alpha$ and $\alpha^{\prime}>\frac{\nu}{p}$, then for a small enough and $d \leq a t^{1 / w}$

$$
\left|p_{t}(x, x)-p_{t}(y, x)\right| \leq \frac{1}{2} p_{t}(x, x),
$$


consequently

$$
\begin{aligned}
p_{t}(x, y) \geq \frac{1}{2} p_{t}(x, x) \geq & \frac{C}{V\left(x, t^{1 / w}\right)}, \\
& \forall x, y \in M, t>0, \text { such that } d(x, y) \leq a t^{1 / w} .
\end{aligned}
$$

Therefore, by Lemma 2 , one concludes that $\left(L E_{w}\right)$ is satisfied.

\subsection{Second characterization.}

Theorem 7. Let $(M, d, \mu, A)$ as above satisfy the doubling condition $\left(D_{\nu}\right)$. Let $w \geq 2$. Assume that $\left(U E_{w}\right)$ holds. Then

$\left(L E_{w}\right) \quad \frac{c}{V\left(x, t^{1 / w}\right)} E_{w}(c, x, y, t) \leq p_{t}(x, y), \quad \forall t>0, x, y \in M$,

implies for all $p$ large enough, there are $\alpha, \alpha^{\prime}>\frac{\nu}{p}$ and $\left.\theta, \theta^{\prime} \in\right] 0,1[$ such that $\alpha-\alpha^{\prime}=\frac{\nu}{p}, \frac{\alpha}{\theta}=\frac{\alpha^{\prime}}{\theta^{\prime}}$ and

$\left(G_{w}\right)\left\{\begin{aligned} \mid f(x) & -f(y) \mid \leq \frac{C}{V^{1 / p}(x, d)} \\ & \times \max \left\{d^{\alpha}\|f\|_{p}^{1-\theta}\left\|A^{\alpha / w \theta} f\right\|_{p}^{\theta}, d^{\alpha^{\prime}}\|f\|_{p}^{1-\theta^{\prime}}\left\|A^{\alpha^{\prime} / w \theta^{\prime}} f\right\|_{p}^{\theta^{\prime}}\right\} \\ \forall f \in \mathcal{D}, x, y \in M . & \end{aligned}\right.$

Conversely, $\left(G_{w}\right)$ for any $p>1, \alpha, \alpha^{\prime}>\frac{\nu}{p}$ and $\left.\theta, \theta^{\prime} \in\right] 0,1[$, implies $\left(L E_{w}\right)$, where d denote to $d(x, y)$.

Proof:

$(\Rightarrow)$ Let $\beta>\frac{\eta}{p^{\prime}}+\frac{\nu}{p}$. Firstly assume that $\beta<w$. For all $f \in \mathcal{D}$, we know that

$$
f=e^{-t A} f+\int_{0}^{t} A e^{-s A} f d s, \quad \forall t>0,
$$

then

(4) $|f(x)-f(y)| \leq\left|e^{-t A} f(x)-e^{-t A} f(y)\right|$

$$
+\int_{0}^{t}\left|A e^{-s A} f(x)-A e^{-s A} f(y)\right| d s
$$

and

$$
\begin{array}{rl}
\int_{0}^{t} \mid A e^{-s A} & f(x)-A e^{-s A} f(y) \mid d s \\
& =\int_{0}^{t}\left|e^{-(s / 2) A} A e^{-(s / 2) A} f(x)-e^{-(s / 2) A} A e^{-(s / 2) A} f(y)\right| d s
\end{array}
$$


Assume that $d \geq t^{1 / w}$. From Proposition 5, we obtain

$$
\begin{aligned}
\int_{0}^{t}\left|e^{-(s / 2) A} A e^{-(s / 2) A} f(x)-e^{-(s / 2) A} A e^{-(s / 2) A} f(y)\right| d s \\
\leq \frac{C}{V^{1 / p}(x, d)} d^{\frac{\eta}{p^{\prime}}+\frac{\nu}{p}} \int_{0}^{t} s^{-\frac{1}{w}\left(\frac{\eta}{p^{\prime}}+\frac{\nu}{p}\right)}\left\|A e^{-(s / 2) A} f\right\|_{p} d s .
\end{aligned}
$$

Since $\left(e^{-t A}\right)_{t>0}$ is analytic, then $\left\|A e^{-(s / 2) A} f\right\|_{p} \leq C s^{\frac{\beta}{w}-1}\left\|A^{\beta / w} f\right\|_{p}$. Hence

$$
\begin{aligned}
\int_{0}^{t} s^{-\frac{1}{w}\left(\frac{\eta}{p^{\prime}}+\frac{\nu}{p}\right)}\left\|A e^{-(s / 2) A} f\right\|_{p} d s & \leq C\left\|A^{\beta / w} f\right\|_{p} \int_{0}^{t} s^{-\frac{1}{w}\left(\frac{\eta}{p^{\prime}}+\frac{\nu}{p}\right)+\frac{\beta}{w}-1} d s \\
& =C t^{-\frac{1}{w}\left(\frac{\eta}{p^{\prime}}+\frac{\nu}{p}\right)+\frac{\beta}{w}}\left\|A^{\beta / w} f\right\|_{p} .
\end{aligned}
$$

Thus

(5) $\int_{0}^{t}\left|A e^{-s A} f(x)-A e^{-s A} f(y)\right| d s$

$$
\leq \frac{C}{V^{1 / p}(x, d)} d^{\frac{\eta}{p^{\prime}}+\frac{\nu}{p}} t^{-\frac{1}{w}\left(\frac{\eta}{p^{\prime}}+\frac{\nu}{p}\right)+\frac{\beta}{w}}\left\|A^{\beta / w} f\right\|_{p} .
$$

If $d \leq t^{1 / w}$.

$$
\begin{gathered}
\int_{0}^{t}\left|A e^{-s A} f(x)-A e^{-s A} f(y)\right| d s=\int_{0}^{d^{w}}\left|A e^{-s A} f(x)-A e^{-s A} f(y)\right| d s \\
+\int_{d^{w}}^{t}\left|e^{-(s / 2) A} A e^{-(s / 2) A} f(x)-e^{-(s / 2) A} A e^{-(s / 2) A} f(y)\right| d s .
\end{gathered}
$$

From (5) and Proposition 5, we obtain

$$
\begin{aligned}
& \int_{0}^{t}\left|A e^{-s A} f(x)-A e^{-s A} f(y)\right| d s \\
\leq & \frac{C}{V^{1 / p}(x, d)} d^{\beta}\left\|A^{\beta / w} f\right\|_{p}+\frac{C}{V^{1 / p}(x, d)} d^{\frac{\eta}{p^{\prime}}} \int_{d^{w}}^{t} s^{-\frac{\eta}{w p^{\prime}}\left\|A e^{-(s / 2) A} f\right\|_{p} d s .}
\end{aligned}
$$

Since $\left(e^{-t A}\right)_{t>0}$ is analytic, then

$$
\begin{aligned}
\int_{d^{w}}^{t} s^{-\frac{\eta}{w p^{\prime}}}\left\|A e^{-(s / 2) A} f\right\|_{p} d s & \leq C\left\|A^{\beta / w} f\right\|_{p} \int_{d^{w}}^{t} s^{-\frac{\eta}{w p^{\prime}}+\frac{\beta}{w}-1} d s \\
& =C\left(t^{-\frac{\eta}{w p^{\prime}}+\frac{\beta}{w}}-d^{-\frac{\eta}{p^{\prime}}+\beta}\right)\left\|A^{\beta / w} f\right\|_{p} .
\end{aligned}
$$


Therefore

$$
\begin{aligned}
& \int_{0}^{t}\left|A e^{-s A} f(x)-A e^{-s A} f(y)\right| d s \\
& \leq \frac{C}{V^{1 / p}(x, d)} d^{\beta}\left\|A^{\beta / w} f\right\|_{p}+\frac{C}{V^{1 / p}(x, d)}\left(d^{\frac{\eta}{p^{\prime}}} t^{-\frac{\eta}{w p^{\prime}}+\frac{\beta}{w}}-d^{\beta}\right)\left\|A^{\beta / w} f\right\|_{p} \\
& (6) \leq \frac{C}{V^{1 / p}(x, d)} d^{\frac{\eta}{p^{\prime}}} t^{-\frac{\eta}{w p^{\prime}}+\frac{\beta}{w}}\left\|A^{\beta / w} f\right\|_{p} .
\end{aligned}
$$

Hence by (4), Proposition 5, (5) and (6), one has for all $t>0$

$$
|f(x)-f(y)| \leq \frac{C}{V^{1 / p}(x, d)} \times(*),
$$

where

$$
\begin{aligned}
(*)=\max \{ & \left(\frac{d}{t^{1 / w}}\right)^{\frac{\eta}{p^{\prime}}+\frac{\nu}{p}}\left(\|f\|_{p}+t^{\beta / w}\left\|A^{\beta / w} f\right\|_{p}\right), \\
& \left.\left(\frac{d}{t^{1 / w}}\right)^{\frac{\eta}{p^{\prime}}}\left(\|f\|_{p}+t^{\beta / w}\left\|A^{\beta / w} f\right\|_{p}\right)\right\} .
\end{aligned}
$$

By choosing $t=\left(\frac{\|f\|_{p}}{\left\|A^{\beta / w} f\right\|_{p}}\right)^{w / \beta}$, one obtains

$$
\begin{aligned}
&|f(x)-f(y)| \leq \frac{C}{V^{1 / p}(x, d)} \\
& \quad \times \max \left\{d^{\frac{\eta}{p^{\prime}}+\frac{\nu}{p}}\|f\|_{p}^{1-\theta}\left\|A^{\beta / w} f\right\|_{p}^{\theta}, d^{\frac{\eta}{p^{\prime}}}\|f\|_{p}^{1-\theta^{\prime}}\left\|A^{\beta / w} f\right\|_{p}^{\theta^{\prime}}\right\},
\end{aligned}
$$

where $\theta=\frac{1}{\beta}\left(\frac{\eta}{p^{\prime}}+\frac{\nu}{p}\right)$ and $\theta^{\prime}=\frac{\eta}{\beta p^{\prime}}$.

Now, if $\beta \geq w$. Fix $k>\frac{\beta}{w}$, we know that

$$
f=\sum_{i=0}^{k-1} \frac{t^{i}}{i !} A^{i} e^{-t A} f+\frac{1}{(k-1) !} \int_{0}^{t} s^{k-1} A^{k} e^{-s A} f d s, \quad \forall t>0 .
$$

By Proposition 5 and the analyticity, it yields, for all $i=0, \ldots, k-1$ :

$$
\begin{aligned}
\left|A^{i} e^{-t A} f(x)-A^{i} e^{-t A} f(y)\right| & \leq \frac{C}{V^{1 / p}(x, d(x, y))}\left\|A^{i} e^{-(t / 2) A} f\right\|_{p} \times(* *) \\
& \leq \frac{C t^{-i}}{V^{1 / p}(x, d(x, y))}\|f\|_{p} \times(* *)
\end{aligned}
$$


for all $t>0, f \in \mathcal{D}$ and $\mu$-a.e. $x, y \in M$. where

$$
(* *)= \begin{cases}\left(\frac{d(x, y)}{t^{1 / w}}\right)^{\frac{\eta}{p^{\prime}}+\frac{\nu}{p}} & \text { if } d(x, y) \geq t^{1 / w} \\ \left(\frac{d(x, y)}{t^{1 / w}}\right)^{\frac{\eta}{p^{\prime}}} & \text { if not. }\end{cases}
$$

On the other hand, one can write

$$
\begin{aligned}
& \int_{0}^{t} s^{k-1}\left(A^{k} e^{-s A} f(x)-A^{k} e^{-s A} f(y)\right) d s \\
& =\int_{0}^{t} s^{k-1}\left(e^{-(s / 2) A} A^{k} e^{-(s / 2) A} f(x)-e^{-(s / 2) A} A^{k} e^{-(s / 2) A} f(y)\right) d s
\end{aligned}
$$

and by analyticity, we have

$$
\left\|A^{k} e^{-(s / 2) A} f\right\|_{p} \leq C s^{\frac{\beta}{w}-k}\left\|A^{\beta / w} f\right\|_{p}
$$

Then, by using Proposition 5 and arguing similarly as the first case, we also obtain

$$
\begin{aligned}
|f(x)-f(y)| \leq \frac{C}{V^{1 / p}(x, d)} \\
\quad \times \max \left\{d^{\frac{\eta}{p^{\prime}}+\frac{\nu}{p}}\|f\|_{p}^{1-\theta}\left\|A^{\beta / w} f\right\|_{p}^{\theta}, d^{\frac{\eta}{p^{\prime}}}\|f\|_{p}^{1-\theta^{\prime}}\left\|A^{\beta / w} f\right\|_{p}^{\theta^{\prime}}\right\},
\end{aligned}
$$

where $\theta=\frac{1}{\beta}\left(\frac{\eta}{p^{\prime}}+\frac{\nu}{p}\right)$ and $\theta^{\prime}=\frac{\eta}{\beta p^{\prime}}$.

Set $\alpha=\frac{\eta}{p^{\prime}}+\frac{\nu}{p}$ and $\alpha^{\prime}=\frac{\eta}{p^{\prime}}$, then $\beta=\frac{\theta}{\alpha}=\frac{\theta^{\prime}}{\alpha^{\prime}}, \alpha-\alpha^{\prime}=\frac{\nu}{p}, \alpha>\frac{\nu}{p}$, $\alpha^{\prime}>0$ and

$$
\begin{aligned}
&|f(x)-f(y)| \leq \frac{C}{V^{1 / p}(x, d)} \\
& \quad \times \max \left\{d^{\alpha}\|f\|_{p}^{1-\theta}\left\|A^{\alpha / w \theta} f\right\|_{p}^{\theta}, d^{\alpha^{\prime}}\|f\|_{p}^{1-\theta^{\prime}}\left\|A^{\alpha^{\prime} / w \theta^{\prime}} f\right\|_{p}^{\theta^{\prime}}\right\} .
\end{aligned}
$$

For $p>\frac{\nu+\eta}{\eta}$, one has $\eta>\frac{\nu+\eta}{p}$, then $\eta\left(1-\frac{1}{p}\right)>\frac{\nu}{p}$, hence $\alpha^{\prime}=\frac{\eta}{p^{\prime}}>\frac{\nu}{p}$.

$(\Leftarrow)$ Let $p>1$, assume that for some $\alpha, \alpha^{\prime}>\frac{\nu}{p}$ and $\left.\theta, \theta^{\prime} \in\right] 0,1[$ :

$$
\begin{aligned}
&|f(x)-f(y)| \leq \frac{C}{V^{1 / p}(x, d)} \\
& \quad \times \max \left\{d^{\alpha}\|f\|_{p}^{1-\theta}\left\|A^{\alpha / w \theta} f\right\|_{p}^{\theta}, d^{\alpha^{\prime}}\|f\|_{p}^{1-\theta^{\prime}}\left\|A^{\alpha^{\prime} / w \theta^{\prime}} f\right\|_{p}^{\theta^{\prime}}\right\},
\end{aligned}
$$

for all $f \in \mathcal{D}$ and $x, y \in M$. 
Let $z \in M$, by analyticity of $\left(e^{-t A}\right)_{t>0}, p_{t}(., z)$ belong to $\mathcal{D}_{p}\left(A^{\alpha / w}\right)$. Then by choosing $f=p_{t}(., z)$ in the previous inequality, one has for all $x, y \in M$ :

$$
\begin{aligned}
\left|p_{t}(x, z)-p_{t}(y, z)\right| \leq & \frac{C}{V^{1 / p}(x, d)} \\
& \quad \times \max \left\{d^{\alpha}\left\|p_{t}(., z)\right\|_{p}^{1-\theta}\left\|A^{\alpha / w \theta} p_{t}(., z)\right\|_{p}^{\theta},\right. \\
& \left.\quad d^{\alpha^{\prime}}\left\|p_{t}(., z)\right\|_{p}^{1-\theta^{\prime}}\left\|A^{\alpha^{\prime} / w \theta^{\prime}} p_{t}(., z)\right\|_{p}^{\theta^{\prime}}\right\} .
\end{aligned}
$$

By analyticity,

$$
\left\|A^{\alpha / w \theta} p_{t}(., z)\right\|_{p}=\left\|A^{\alpha / w \theta} e^{-(t / 2) A} p_{t / 2}(., z)\right\|_{p} \leq C t^{-\alpha / w \theta}\left\|p_{t / 2}(., z)\right\|_{p} .
$$

Since $\left(e^{-t A}\right)_{t>0}$ is symmetric Markovian, then $\left\|p_{t / 2}(., z)\right\|_{1} \leq 1$. Hence $\left(U E_{w}\right)$ yields

$$
\left\|p_{t}(., z)\right\|_{p} \leq \frac{C}{\left[V\left(z, t^{1 / w}\right)\right]^{1-\frac{1}{p}}}, \quad \forall t>0,
$$

and by $\left(D_{\nu}\right)$, one has

$$
\left\|p_{t / 2}(., z)\right\|_{p} \leq \frac{C}{\left[V\left(z, t^{1 / w}\right)\right]^{1-\frac{1}{p}}}
$$

then

$$
\left\|p_{t}(., z)\right\|_{p}^{1-\theta}\left\|A^{\alpha / w \theta} p_{t}(., z)\right\|_{p}^{\theta} \leq C \frac{t^{\alpha / w}}{\left[V\left(z, t^{1 / w}\right)\right]^{1-\frac{1}{p}}} .
$$

Therefore

$$
\begin{aligned}
& \left|p_{t}(x, z)-p_{t}(y, z)\right| \\
& \leq \frac{C}{V^{1 / p}(x, d)} \max \left\{\left(\frac{d}{t^{1 / w}}\right)^{\alpha} \frac{1}{\left[V\left(z, t^{1 / w}\right)\right]^{1-\frac{1}{p}}},\left(\frac{d}{t^{1 / w}}\right)^{\alpha^{\prime}} \frac{1}{\left[V\left(z, t^{1 / w}\right)\right]^{1-\frac{1}{p}}}\right\} \\
& \leq \frac{C}{V\left(z, t^{1 / w}\right)}\left(\frac{V\left(z, t^{1 / w}\right)}{V(x, d)}\right)^{1 / p} \max \left\{\left(\frac{d}{t^{1 / w}}\right)^{\alpha},\left(\frac{d}{t^{1 / w}}\right)^{\alpha^{\prime}}\right\} .
\end{aligned}
$$

Then, similarly as in the proof of the converse in Theorem 6 , we obtain the desired result.

A consequence of Theorems 6 and 7 is the following. 
Corollary 8. Let $(M, d, \mu, A)$ as above satisfy the doubling condition $\left(D_{\nu}\right)$. Assume that $\left(U E_{w}\right)$ holds. Then

$$
\left(S_{w}\right) \text { for any } p>1 \text { and } \alpha, \alpha^{\prime}>\frac{\nu}{p},
$$

implies, for all $p$ large enough, that there are $\alpha, \alpha^{\prime}>\frac{\nu}{p}$ and $\left.\theta, \theta^{\prime} \in\right] 0,1[$ such that $\alpha-\alpha^{\prime}=\frac{\nu}{p}, \frac{\alpha}{\theta}=\frac{\alpha^{\prime}}{\theta^{\prime}}$ and $\left(G_{w}\right)$ holds.

Conversely, $\left(G_{w}\right)$ for any $p>1$ and $\left.\alpha, \alpha^{\prime}>\frac{\nu}{p}, \theta, \theta^{\prime} \in\right] 0,1[$, implies, for all $p$ large enough, that there are $\alpha, \alpha^{\prime}>\frac{\nu}{p}$ such that $\left(S_{w}\right)$ holds.

Remark 9 . We can also deduce $\left(G_{w}\right)$ from $\left(S_{w}\right)$ by using the momentum inequality: for a nonnegative operator in a Banach space

$$
\left\|A^{\alpha / w} f\right\|_{p} \leq C\|f\|_{p}^{1-\theta}\left\|A^{\alpha / w \theta} f\right\|_{p}^{\theta}
$$

(see $[\mathbf{1 5}]$ ). The previous corollary, gives us more information about the relation between $\alpha, \alpha^{\prime}, \theta$ and $\theta^{\prime}$.

\subsection{Further results.}

Let $(M, d, \mu, A)$ be as before and $w \geq 2$. From the doubling property, one can also write, there are $\nu>0$ and $\nu^{\prime} \geq 0$ such that

$$
\left(D_{\nu, \nu^{\prime}}\right) \quad C^{\prime}\left(\frac{s}{r}\right)^{\nu^{\prime}} \leq \frac{V(x, s)}{V(x, r)} \leq C\left(\frac{s}{r}\right)^{\nu}, \quad \forall x \in M, s \geq r>0 .
$$

Note that, when $M$ has an infinite measure, we can take $\nu^{\prime}>0$, (see [8]).

By using $\left(D_{\nu, \nu^{\prime}}\right)$ instead of $\left(D_{\nu}\right)$ and arguing as before, almost all the previous results can be written for all $p>1$ with $\nu$ and $\nu^{\prime}$. In other words, one has just take $\nu^{\prime}=0$ (which is always possible) to find the previous proofs.

More precisely, instead of Propositions 4 and 5, we obtain: If $\left(L Y_{w}\right)$ holds, then there exists $\eta \in] 0,1[$ such that for all $p>1$ :

$$
\begin{aligned}
& \left|p_{t}(x, z)-p_{t}(y, z)\right| \\
& \leq C \frac{\left(E_{w}(C, x, z, t)+E_{w}(C, y, z, t)\right)^{1 / p}}{V^{1 / p}(x, d) V^{1 / p^{\prime}}\left(z, t^{1 / w}\right)} \begin{cases}\left(\frac{d}{t^{1 / w}}\right)^{\frac{\eta}{p^{\prime}}+\frac{\nu}{p}} & \text { if } d \geq t^{1 / w} \\
\left(\frac{d}{t^{1 / w}}\right)^{\frac{\eta}{p^{\prime}}+\frac{\nu^{\prime}}{p}} & \text { if not }\end{cases}
\end{aligned}
$$

for all $t>0$ and $\mu$-a.e. $x, y, z \in M$. 
And

(7)

$\left|e^{-t A} f(x)-e^{-t A} f(y)\right| \leq \frac{C\|f\|_{p}}{V^{1 / p}(x, d)} \begin{cases}\left(\frac{d}{t^{1 / w}}\right)^{\frac{\eta}{p^{\prime}}+\frac{\nu}{p}}, & \text { if } d \geq t^{1 / w} \\ \left(\frac{d}{t^{1 / w}}\right)^{\frac{\eta}{p^{\prime}}+\frac{\nu^{\prime}}{p}} & \text { if not, }\end{cases}$

for all $t>0, f \in \mathcal{D}$ and $\mu$-a.e. $x, y \in M$, where $d$ denote to $d(x, y)$.

Consequently, we obtain the following proposition.

Proposition 10. Let $(M, d, \mu, A)$ as before satisfy the doubling condition $\left(D_{\nu, \nu^{\prime}}\right)$, assume that the $\left(U E_{w}\right)$ holds, then for all $p>1$,

$$
\begin{aligned}
\text { (i) } \quad\left(L E_{w}\right) \Rightarrow\left(S_{w}\right) \text { for some } \alpha>\frac{\nu}{p} \text { and } \alpha^{\prime}>\frac{\nu^{\prime}}{p} . \\
\text { (ii) } \quad\left(L E_{w}\right) \Rightarrow\left(G_{w}\right) \text { for some } \alpha>\frac{\nu}{p}, \alpha^{\prime}>\frac{\nu^{\prime}}{p} \\
\text { such that } \alpha-\alpha^{\prime}=\frac{\nu-\nu^{\prime}}{p} \text { and } \frac{\alpha}{\theta}=\frac{\alpha^{\prime}}{\theta^{\prime}} .
\end{aligned}
$$

Proof: (i) By using (7) instead of Proposition 5 in the proof of Theorem 6 , we obtain for all $p>1,\left(S_{w}\right)$ holds for $\alpha=\frac{\eta}{p^{\prime}}+\frac{\nu+\delta}{p}$ and $\alpha^{\prime}=\frac{\eta}{p^{\prime}}+\frac{\nu-\delta}{p}$ where $\left.\delta \in\right] 0, \frac{p}{p^{\prime}} \eta\left[\right.$, whence $\alpha>\frac{\nu}{p}$ and $\alpha^{\prime}>\frac{\nu^{\prime}}{p}$.

(ii) Similarly as in the proof of Theorem 7 and by using (7) instead of Proposition 5, we obtain that for all $p>1$, there are $\alpha=\frac{\eta}{p^{\prime}}+\frac{\nu}{p}$, $\left.\alpha^{\prime}=\frac{\eta}{p^{\prime}}+\frac{\nu^{\prime}}{p}, \theta, \theta^{\prime} \in\right] 0,1\left[\operatorname{such}\right.$ that $\frac{\alpha}{\theta}=\frac{\alpha^{\prime}}{\theta^{\prime}}$ and $\left(G_{w}\right)$ holds. It is clear that $\alpha-\alpha^{\prime}=\frac{\nu-\nu^{\prime}}{p}$.

In the case when the volume growth is polynomial (i.e. $V(x, r) \simeq r^{D}$, $D>0$ ), we reobtain the following result from Ouhabaz (see [16]).

Corollary 11. Assume that $V(x, r) \simeq r^{D}$, if $\left(U E_{w}\right)$ holds, then for all $p>1$

$$
\begin{aligned}
& \left(L E_{w}\right) \Leftrightarrow \frac{\mid f(x)-f(y)}{d(x, y)^{\alpha-\frac{D}{p}}} \leq C\|f\|_{p}^{\theta}\left\|A^{\alpha / w \theta} f\right\|_{p}^{1-\theta}, \\
& \left.\quad \text { for some } \alpha>\frac{D}{p}, \theta \in\right] 0,1[.
\end{aligned}
$$


Proof: If $V(x, r) \simeq r^{D}$, then one can write $\left(D_{\nu, \nu^{\prime}}\right)$ with $\nu=\nu^{\prime}=D$. Therefore

$(\Leftarrow)$ it suffices to take $\alpha=\alpha^{\prime}$ and use Theorem 7 .

$(\Rightarrow)$ by applying (ii) of the previous proposition, $\left(L E_{w}\right)$ implies $\left(G_{w}\right)$ with $\alpha=\alpha^{\prime}>\frac{D}{p}$ and $\theta=\theta^{\prime}$.

Acknowledgements. I would like to thank Professor Thierry Coulhon, who brought the subject to my attention, for interesting and stimulating discussions. My thanks go also to Professor El-Maati Ouhabaz, for interesting conversations and suggestions during our meeting at the colloquium "Analyse Fonctionnelle et Harmonique, 19-22 Novembre 07", held at the CIRM "Centre International des Rencontres Mathématiques". I would also like to thank the referees for useful comments.

\section{References}

[1] M. T. BARLOW, Which values of the volume growth and escape time exponent are possible for a graph?, Rev. Mat. Iberoamericana 20(1) (2004), 1-31.

[2] M. T. BARLOW AND R. F. BASS, Random walks on graphical Sierpinski carpets, in: "Random walks and discrete potential theory" (Cortona, 1997), Sympos. Math. XXXIX, Cambridge Univ. Press, Cambridge, 1999, pp. 26-55.

[3] M. T. BARlow and R. F. BAss, Divergence form operators on fractal-like domains, J. Funct. Anal. 175(1) (2000), 214-247.

[4] I. Benjamini, I. Chavel, and E. A. Feldman, Heat kernel lower bounds on Riemannian manifolds using the old ideas of Nash, Proc. London Math. Soc. (3) 72(1) (1996), 215-240.

[5] T. Coulhon, Off-diagonal heat kernel lower bounds without Poincaré, J. London Math. Soc. (2) 68(3) (2003), 795-816.

[6] T. Coulhon, Ultracontractivity and Nash type inequalities, $J$. Funct. Anal. 141(2) (1996), 510-539.

[7] T. Coulhon and A. Grigor'yan, Random walks on graphs with regular volume growth, Geom. Funct. Anal. 8(4) (1998), 656-701.

[8] A. GRIGOR'YAN, The heat equation on noncompact Riemannian manifolds, (Russian), Mat. Sb. 182(1) (1991), 55-87; translation in: Math. USSR-Sb. 72(1) (1992), 47-77.

[9] A. Grigor'Yan, Heat kernel upper bounds on a complete non-compact manifold, Rev. Mat. Iberoamericana 10(2) (1994), 395-452. 
[10] A. Grigor'Yan, J. Hu, and K.-S. LaU, Heat kernels on metric measure spaces and an application to semilinear elliptic equations, Trans. Amer. Math. Soc. 355(5) (2003), 2065-2095 (electronic).

[11] A. GRigor'Yan AND A. Telcs, Sub-Gaussian estimates of heat kernels on infinite graphs, Duke Math. J. 109(3) (2001), 451-510.

[12] B. Hambly and T. Kumagai, Heat kernel estimates for symmetric random walks on a class of fractal graphs and stability under rough isometries, in: "Fractal geometry and applications: a jubilee of Benoit Mandelbrot, Part 2", Proc. Sympos. Pure Math. 72, Amer. Math. Soc., Providence, RI, 2004, pp. 233-259.

[13] W. Hebisch and L. Saloff-Coste, On the relation between elliptic and parabolic Harnack inequalities, Ann. Inst. Fourier (Grenoble) 51(5) (2001), 1437-1481.

[14] P. Li AND S.-T. YAU, On the parabolic kernel of the Schrödinger operator, Acta Math. 156(3-4) (1986), 153-201.

[15] S. Martines, M. Sans, And M. D. Martines, Some inequalities for fractional integrals and derivatives, (Russian), Dokl. Akad. Nauk SSSR 315(5) (1990), 1049-1051; translation in: Soviet Math. Dokl. 42(3) (1991), 876-879.

[16] E.-M. Ouhabaz, Heat kernels of multiplicative perturbations: Hölder estimates and Gaussian lower bounds, Indiana Univ. Math. J. 47(4) (1998), 1481-1495.

[17] E.-M. OuhabaZ, "Analysis of heat equations on domains", London Mathematical Society Monographs Series 31, Princeton University Press, Princeton, NJ, 2005.

[18] L. Saloff-Coste, A note on Poincaré, Sobolev, and Harnack inequalities, Internat. Math. Res. Notices 2 (1992), 27-38.

[19] L. SAloff-Coste, Parabolic Harnack inequality for divergenceform second-order differential operators, Potential theory and degenerate partial differential operators (Parma), Potential Anal. 4(4) (1995), 429-467.

[20] L. Saloff-Coste, "Aspects of Sobolev-type inequalities", London Mathematical Society Lecture Note Series 289, Cambridge University Press, Cambridge, 2002.

[21] E. M. Stein, "Topics in harmonic analysis related to the Littlewood-Paley theory", Annals of Mathematics Studies 63, Princeton University Press, Princeton, N.J.; University of Tokyo Press, Tokyo, 1970. 
Département de Mathématiques

Université de Cergy-Pontoise

Site de Saint Martin, 2 avenue Adolphe-Chauvin

95302 Cergy-Pontoise cedex

France

E-mail address: salahaddine.boutayeb@u-cergy.fr

Primera versió rebuda el 29 d'abril de 2008,

darrera versió rebuda el 18 de setembre de 2008. 